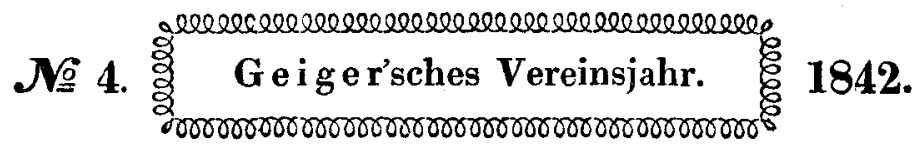

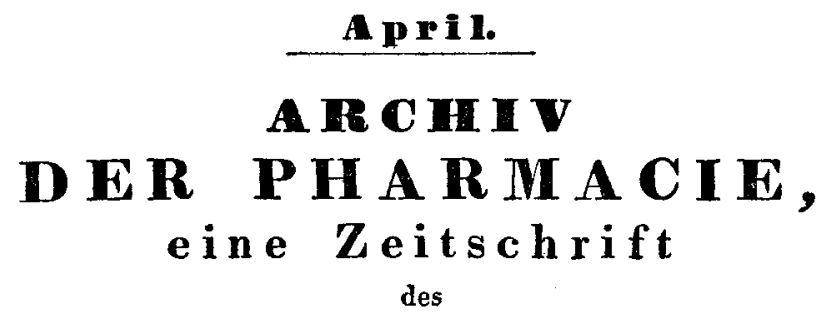

A potheker-Vereins in Norddeutschland.

Zweite Reihe. Dreifsigsten Bandes erstes Heft.

\title{
Erste Abtheilung.
}

\section{Vereinszeitung,}

redigirt vom Directorio des Vereins.

\section{1) Biographische Denkmäler.}

Nän e und $\mathbf{n}$ ach ect

dem

Andenken meines verstorbenen wissenschaftlichen Freundes, des Apothekers Dr. Fr. Sertürner in Hameln, gewidmet;

von

Dr. E. Nolte in Hannover.

Wenn es mir gleich nicht gestattet ist, in den folgenden kurzen biographischen Notizen einen vollständigen speciellen Nekrolog des, der Wissenschaft zu frïh entrissenen ausgezeichneten Forschero im Felde der Chemie und höhern Physik aufzustellen, so kann ich doch nicht umhin, dem Zuge meines Herzens zu folgen, und meinem verstorbenen Freunde und der Wissenschaft ein Todtenopfer zu bringen, welches für Mit- und Nachwelt die grofsen Verdienste des so vielfach verkannten Verstorbenen im Fache der allgemeinen und besondern Naturwissenschaft nach ihrem vollen Werthe würdigt, und bleibend erhalten wird, trotz so manchen, das Innere tief verletzenden Ver-

Arch. d. Pharm. II. Reihe. XXX. Bds. 1. Hft. 
unglimpfungen und ungerechten bittern Tadels einiger Gegner in frühern Zeiten, die er im Leben erlitt, und welche wahrscheinlich den ersten unvertilgbaren Keim des frïhen Todes in den Gesammtorganismus desselben versenkte. Nur der unpartheiische Freund der Wissenschaft wird mit mir den vielleicht zu spät gefeierten Triumph der Wahrheit gehörig würdigen, und den Manen des Verewigten ein bleibendes ehrendes Andenken weihen.

Es war gewifs eine unglïckliche Folge der vielfachen unverdienten Kränkungen, die der zu früh Dahingeschiedene in seinem vielbewegten, emsig forschenden Leben erleiden mufste, dafs seine an sich kräftige Natur seit einigen Jahren einen schwankenden Gesundheitszustand erlitt, welcher psychisch und somatisch für nahe oder entfernte Zukunft vielseitige Besorgnisse erregte, die leider auch in der jüngsten Zeit sich zunächst in successiv aggravirter hypochondrischer Gemuithsstimmung und unverkennbarer stiller Seelenstörung aussprach, und im Verlaufe der Zeit sich allgemeine Gichtbeschwerden entwickelten, welche durch Hartnäckigkeit eine tief gewurzelte Grundlage deutlich zeigten. Nach vielfältig angewandten pharmaceutischen Heilversuchen, die aber die schmerzhaften arthritischen Beschwerden nicht bleibend beseitigten, unternahm der hart geprüfte Kranke im Sommer 1839 eine Badekur in Wiesbaden, von woher er zwar nach sechswöchentlicher Brunnen- und Badekur merklich gebessert zurückkehrte, aber erst lange Zeit nachher, bei fortgesetzter pharmaceutischer Procedur der Austilgung der arthritischen Dyscrasie sich erfreuen durfte.

Diese lang gedauerte schmerzhafte gichtische Affection machte ihn nicht nur zu allen Geschäften und wissenschaftlichen Untersuchungen gänzlich unfähig, sondern gab auch Veranlassung zu Unterbrechung unserer Correspondenz, bis ich endlich Gelegenheit hatte, mich gegen Ende 1840 von dem damaligen Wohlbefinden meines Freundes, dem ich so manche Belehrung im Fache der physikalischen Chemie und Pharmacie, so wie der Heilkunde verdanke, persönlich zu iberzeugen.

Allein noch während seines kurzen hiesigen Aufenthalts wurde er unvermuthet in der Nacht wegen gewisser Familienangelegenheiten nach Eimberk ahgerufen, woselbst er bei ungestörter Gesundheit bis Anfangs Februar dieses Jahrs verweilte, und darauf nach Hameln wieder zurückkehrte. Bis zum 10. d. M. genofs er nun eines anscheinend ungestörten Wohlbefindens, hatte zu Mittag mit gutem Appetit gegessen, besuchte Nachmittags einen dortigen Club, und trank am Abend im Kreise seiner Familie eine Tasse Thee, wurde aber nach dem Genusse einer zweiten Tasse plötzlich von allgemeinem Froste befallen, und konnte nur mit Unterstiitzung zu Bett gebracht werden. Aber schon nach zwei Stunden brachen Phantasien aus, wefshalb sein Arzt zu Huilfe gerufen wurde, der aufser andern Arzneien mit allgemeinen und örtlichen Blutentziehungen einschritt, obwohl ohne gïnstigen Erfolg, denn die Phantasien dauerten unaufgehalten fort, und der Kranke kam gar nicht zur Besinnung. Wegen anhaltender Schlaflosigkeit wurde im fernern Verlaufe Opium gereicht, allein ohne wohlthätige Folgen, da nur vorübergehende Ruhe erfolgte, und die erneuerten Phantasien nicht nur anhal- 
tend fortdauerten, sondern bereits am sechsten Tage Zeichen des herannahenden Todes bemerkt wurden, welcher auch am Abend des neunten Tages unter schrecklichen Krämpfen erfolgte.

In Folge der brieflichen Mittheilung eines Mitgliedes der Familie sollten die Aerzte die Vermuthung aufgestellt haben, dafs wahrscheinlich ein Ergufs von Wasser in die Höhle des Rijckenmarks und eine Infiltration von $W$ asser ins Gehirn den Grund zu dieser schnell tödtlichen Krankheit gelegt haben minsse, welche Vermuthung auch durch den Umstand noch mehr bekräftigt worden sei, dafs der ganze Habitus des Körpers einen wassersühtigen Anschein gehabt habe. Fine leider unterbliebene Section des Leichnams würde diese diagnostische Vermuthung bestaitigt haben.

So endete ein Mann auf einer solchen Lebensstufe, am 20. Febr. 1841, in seinem 58. I,ebensjahre, die einen so frïhen Verlust nicht ahnen liefs, ein Mann, dessen grofse Verdienste um die Wissenschaft zum Theil vielfach anerkannt wurden, obgleich auch von andern Seiten sein wissenschaftlicher Werth häufig bekrittelt und verdientermalsen nicht gewiirdigt wurde. Die Ruhe im Grabe gleicht übrigens alle bifferenzen und Widerspruiche seiner Gegner im Leben aus, ohne den Nachruhm zu schmälern.

Wir finden es nun in der täglichen Erfahrung bestätigt, dars jedes geistige Talent im Menschen früh sich zu entwickeln pflegt, und der Mensch in späterer Zeitfolge zu einer Höhe der geistigren Vollkommenheit und Virtuosität gelangt, die von dem fortschreitenden Streben des Geistes hin und wieder auf Kosten des materiellen Lebens zeugt. Dies war auch bei unserm trefflichen Sertürner der Fall, indem ein angebornes Talent ihm den scientifischen $W$ eg zeigte, auf welchem fortschreitend er den ersten Grund zu dem nachher erlangten gelehrten Rufe legte.

Beharrliches Streben im Felde der Chemie und Pharmacie, wie solches sein Fach als Pharmaceut darbot, gab zunächst Veranlassung zu der wichtigen Entdeckung des wesentlichen Grundstoffs und des wirkenden Princips des opiums, welches er zum Gegenstande seiner chemischen Untersuchung gewählt hatte, in der Eigenschaft einer alkalischen Salzbase, von ihm Morphium genannt, womit er zuerst die chemisch-pharmaceutische $W$ issenschaft und die Heilkunde bereicherte, und zugleich der Frste war, der die Bahn zur nachherigen Entdeckung mehrer wichtiger giftiger Alkaloiden, als die Grundstoffe verschiedener vegetabilischer Natur - und Arzneikörper brach, die späterhin, zunächst von mehren ausgezeichneten französischen Chemikern, Pelletier, Caventou, Lassaigne, Magendie elc., unter welchen auch Derosne, gleichzeitig mit Sertürner, das Opium zum Gegenstande seiner chemischen Untersuchung gewählt hatte, entdeckt wurden, denen sich auch unsere deutschen Chemiker, Pf aff, Brandes, Geiger u. a. anschlossen. Diese alkalischen Salzbasen, unter welchen ich nur des Chinins, Brucins, Veratrins und Strychnins zu erwähnen brauche, sind hinlänglich als kräftige Arzneikörper bekannt.

Die erste Entdeckung des Alkaloids des Opiums fiel in die frühe Jugendzeit Sertürner's, als er 1804 in der Apotheke in 
Eimbeck servirte, und sprach also für die grofsen chemischpharmaceutischen Kenntnisse des wackern jungen Mannes, und der tief forschende Geist desselben bewährte sich im Laufe der Zeit in solchem Maafse, dafs er trotz allem Widerspruch und Tadel der Gegner, unter welchen auch Berzelius auftrat, welcher grofse Chemiker sich aber später mit Sertürner verständigte, - nicht ermüdete, die angefangenen Untersuchungen in Mufsestunden fortzusetzen, um endlich zu einem, seine Forschungen krönenden Resultate zu gelangen, wie er solches in Gilberi's Annalen der Physik, 25. B. 1817. Heft 1. p. $56-89$ in einem weitlüuftigen Aufsatze öffentlich dargelegt hat.

Welcher wissenschaftlich gebildete Kunstverständige wird in dieser Abhandlung den Meister in der physikalisch-chemischen Wissenschaft, so wie insbesondere der analytischen Chemie, auch in ihren feinsten chemischen Beziehungen und Verwandtschaften verkennen?

Da nun aber gleichzeitig mit Sertiirner Hr. Derosne in Paris ebenfalls eine chemische Untersuchung des Opiums anstellte, und in Folge derselben die Ehre der ersten Entdeckung des Grundprincips des Opiums für sich vindicirte, sich aber später durch eine von Sertürner wiederholte chemische Analyse des Opiums erwies, dafs der von Derosne dargestellte Grundstoff nicht Morphium war, so sah sich die Pariser Akademie der Wissenschaften veranlafst, einen Preis von 2000 Franken für denjenigen Chemiker auszusetzen, dem nach dem Ausspruche der Akademie die Ehre der ersten Entdeckung des reinen Morphiums zuerkannt werden müsse, und derselbe demnach des Preises würdig sein würde. Die Stimmen der Akademiker waren nun für Sertürner gïnstig, so dafs ihm der Preis zuerkannt und späterhin ausgezahlt wurde. Diese Auszeichnung war um so ehrender für Sertiurner, als er mit einem ausgezeichneten französischen Chemiker, wie Hr. Derosne, um den wissenschaftlichen Vorrang stritt, der ihm auch ohne alle Partheilichkeit, als Sieg der unbestreitbaren Wahrheit, zuerkannt wurde.

Durch diese erste gelungene Arbeit legte nun Sertürnar den Grund zu seiner nachherigen anerkannten gelehrten Auszeichnung im Fache der höhern physikalischen Chemie, die sich in mehren seiner nachherigen Schriften ausspricht. Zunächst erschien von ihm ein, mit grofsem Scharfsinn ausgearbeitetes Werk, wenn auch hin und wieder auf hypothetische Prämissen gestützt, unter folgendem Titel: System der chemischen Physik, 2 Bde. Göttìng. $1820 u .1822$. - Durch dieses höchst wichtige Werk hat sich Sertürner das grofse Verdienst erworben, seine physikalisch-chemischen Untersuchungen da anzureihen, wo der berühmte französische Chemiker La vo is ier aufzuhören gezwungen war, indem dieser als Generalpächter die Wuth der Revolutionsmänner auf sich leitete, und unter dem Messer der Guillotine fiel. Lavoisier war nahe daran, die gewichtlosen Stoffe in der Natur und ihre grolsen Kräfte mit den wägbaren in gegenseitige Verbindung zu setzen, und den nähern wicktigen Zusammenhang zu erkennen, worin die ponderabeln Elemente mit den imponderabeln stehen. Allein durch dieses gewaltsame blutige Ereignifs entging ihm gerade die wichtige Rolle, welche der. Sauerstoff und seine vielfache 
Verwandtschaft und Verbindung mit imponderabeln Stoffen an die Hand giebt. Dieses grofse Verdienst war nun aber unserm S ertürnèr vorbehalten, und jeder unpartheiisehe Leser wird in diesem Werke den tief forschenden Geist Sertïrner's erkennen, nur schade, dafs hin und wieder seine Sprache nicht so klar und deutlich ausgedrïckt ist, um allgemein verstanden $\mathrm{zu}$ werden, um so mehr, als manche tief durchdachte Gegenstände im hypothetischen Gewande erscheinen. Doch wer mag es läugnen, dafs in dem grofsen Felde der Naturwissenschaften Räthsel auf Räthsel sich häufen, die sich nur auf Hypothesen gründen? Ich brauche ja nur des sublimen Gebiets der Astronomie zu erwähnen. Ist dies nicht auch der Fall im Bereiche der physikalischen Chemie, zumal wo es sich um das Substract der hehren allgemeinen Weltkräfte der Imponderabilien handelt, ohnè welche alles Wägbare in der Natur in todter Ruhe verharren wïrde?

In einem besondern Abschnitte des obigen Werks zieht nun Sertürner auch die grofsen allgemeinen Weltkräfte, Licht, Wärme, Electricität und Magnetismus, in Beziehung zum animalischen Leben, wenn auch nur in summarischen Zügen, in den Kreis sciner Untersuchungen, indem er zu erweisen sucht, dafs das organische Gesammtleben in seinen Uranfängen durch den Einflufs jener Quadrupel - Allianz der gewichtlosen Urstoffe der grofsen Weltkräfte, auf erster Stufe entzündet, in fortschreitender Thätigkeit und Ausdauer nach allgemeinen animalischen Naturgesetzen erhalten wird, so lange keine dieser allgemeinen Weltkräfte durch irgend eine äufsere oder innere Bedingung die Oberhand gewinnt, die Ordnung im normalen Gange des Lebensprocesses stört, und Abnormität in dem naturgemärsen Fortgang derselben hervorruft.

Nach diesen allgemeinen Andeutungen des Uranfangs des animalischen Lebensprocesses trägt nun unser Sertỉ rner eine bescheidene Scheu, - wie er pag. 221 des obigen $W$ erkes anführt, - tiefer in das Speciellere der Functionen des organischen Lebeas einzudringen, wie selbige, durch die Imponderabilien von vorn herein zuerst angeregt, sich gegenseitig unterstützen, weil er fürchtet, aus Unkunde der vielfachen sphärischen Verflechtungen von Nerven, Gefälsen und Muskeln Fehlgriffe zu thun, da er den wechselseitigen Zusammenhang dieser einzelnen Theile und ihrer besondern Functionen praktisch zu wenig kerne. Nur so viel stehe fest, dal's im höhern thierischen Organismus die Lungen, oder die Stellvertreter derselben, die Quelle bilden, wo dem thierischen Körper die ersten Lebensgeister, die Electricitöten, - vorzüglich die gevichtige Grundlage des Sauerstoffgases, zugeführt werden, um sich mit den wägbaren Stoffen des Körpers zum Leben, und dessen Fortdauer zu verbinden. (Nur während des Foetuslebens ist der Fall anders, denn der Foetus führt noch kein Eigenleben, sondern ist, abhängig vom Leben der Mutter, nur als Pflanze zu betrachten, und das Leben desselben steht noch auf der niedern Stufe des vegetativen Lebensprocesses, der bekanntlich seinen centralen Heerd, als das vegetative Leben anregend, in der Leber findet, während das selbstständige animale Leben nur in automatischen Bewegungen ausgedrïck ist). 
Von diesen vorbenannten Grundsätzen ging nun Sertür $\mathbf{n}$ er aus, um darauf in mehren Heften seines folgenden periodischen Werkes seine Untersuchungen und Erfahrungen zu begründen:

Die neuesten Entdeckungen in der Physik, Heilkunde und Chemie, auch unter dem besondern Titel: Annalen für das Universalsystem der Elemente, 2 Bde., und 3. Bds. 1. u. 2. Heft. Götling. $1826-1829.8$.

von dahin aber leider! nicht fortgesetzt, wahrscheinlich seiner nachherigen Kränklichkeit wegen.

In diesem neuern periodischen Werke spricht sich nun Sertürner in verschiedenen interessanten Aufsätzen nicht nur als tiefen Forscher im Gebiet der allgemeinen physikalischen Chemie aus, sondern auch insbesondere im Felde der Pharmacie und Heilkunde zeigte sich sein Genie von einer glänzenden Seite, um gerechten Anspruch auf bleibendes Verdienst zu machen. Unter anderm haben wir ihm, - um vorläufig nur eines Gegenstandes zu erwähnen, - ein verbessertes pharmaceutisches Hleipräparat zu verdanken, bei dessen äufserep Anwerdung die Heilkunde uberall nichts von den sonstigen giftigen Eigenschaften des heimtuckischen Bleis zu fürchten hat. Es ist dies ein, in den Säften des thierischen Körpers unauflösliches Bleioxydhydrat, und ein damit bereitetes Bleiwasser, statt des leicht auflöslichen Bleiextracts, dem kohlensauren Blei - Bleiweirs - und dem essigsauren sogenannten Goulard'schen Wasser, wo der äursere Gebrauch so leicht höchst gefahrliche Folgen bereitet. Das Nähere äber die Bereitung des obigen neuern Bleipräparats sehe man in den oben bemerkten Annalen etc. 2. Bd. 2. H. pag. 196 - 228, wo mit Beifigung einiger beweisender Krankheitsfälle weitläuftiger über die bisher gebräuchlichen äufserlichen Bleimittel gehandelt wird. Möchten doch meine eignen, durch mehrjährigen Gebrauch dieses schätzbaren, gänzlich gefahrlosen aufsern Bleimittels erprobten Erfahrungen etwas dazu beitragen, um den Gebrauch desselben allgemeiner zu machen, und durch gänzliche Verbannung der von Aerzten, Wundärzten und Laien noch immer in Anwendung gezogenen schädlichen und gefährlichen Bleipräparate das Verdienst und das Andenken Sertiirner's noch im Tode zu ehren und bleibend zu erhalten!

Aber das so eben Angeregte wäre doch eins der geringern Verdienste des theuren Verstorbenen, hätte ex nicht noch gröfsere, und für allgemeines Menschenwohl wichtigere Ansprüche machen können, die aber auch von so vielen Seiten nicht anerkannt und gewürdigt wurden, durch dringende Empfehlung der Erden und Alkalien, zur Verhütung und Heilung grofser und gefährlicher Krankheiten, die sowohl in kindlichem als fortgeriicktem Alter das Leben des INenschen bedrohen, und vielfach austilgen.

Vorzüglich wichtig ist in dieser Hinsicht der Inhalt des 1. und 2. Hefts des 3. Bandes der Annalen, worin sich der scharf prüfende Geistesblick Sertürner's so hell, und mit der Natur consequent ausspricht. Zunächst und im allgemeinen UJeberblick handelt derselbe über die schädlichen Producte der Verdauung in den meisten Krankheitsperioden, und deren mannichfachen verheerenlen Folgen, einem Gegenstande, der ohne Widerrede tief und hin und wieder verderblich ins allgemeine gesunde Menschen- 
leben eingreift, und als ursächliche Potenz von vorn herein und auf erster ursprïnglicher Stufe so manche wichtige und gefährliche Krankheitsformen bedingt. Welcher rationell handelnde praktische Arzt wird nicht mit dieser Ansicht S ertürner's dahin einverstanden sein, dafs aufser den unvermeidlichen störenden Einflüssen, welche einestheils die Imponderabilien, anderntheils dis rein materiellen, klimatischen, durch Wechsel der Jahrszeiten, der Lufttemperatur, Trockenheit und Feuchtigkeit der Atınosphäre, miasmatische, contagiöse oder nichtcontagióse Ausdüntungen, als endemische oder epidemische Zündstoffe, auf den menschlichen Körper ausüten, auch schädliche Nahrungsstoffe, und dem zufolge fehlerhafte Absonderung eines gesunden Nahrungssaftes, so wie demgemäls eine dyscrasische Mischung des Bluts und der übrigen Säfte des Körpers, den Grund zu einem Heere von Krankheiten lezen, die sämmtlich ursprünglich in den schädlichen Producten der Verdauung zu finden sind? Sehen wir nicht aus dieser Ursache, plötzlich oder allmälig, Unordnungen und gröfsere Störungen in so manchen wichtigen Lebensfunctionen entstehen, die sich zunächst in Unordnungen der Ab- und Aussonderungen, Störung der Erslust, des Schlafs, der Gemüthsstimmung etc. kund geben? - Und alles dies beruht ursprïglich auf fehlerhafter Function des Magens und Darmkanals, wenn der sonst milde Nahrungssaft mit schädlichen, gewöhnlich sauren oder scharfen Stoffen iberladen wird, und weiterhin, ins Blut übergeführt, nicht selten stïrmische und gefährliche Affectionen, zum Theil im Blute selbst, erregt werden, wenn das mit scharfer Säure überladene Blut zunächst die Gefäfse selbst reizt, fieberhafte Regungen veranlafst, und dieser scharfe Stoff bis zur Haut fortgepfianzt, oder auf Schleim-oder andere Häute abgelagert, in acuten exanthematischen Krankheiten, Gicht, Rheumatismus etc. seine oft verderbliche. Rolle spielt.

Als minder turbulent erscheinen nun aber zu Zeiten die Folgen einer regelwidrigen Verdauung nicht nur in den ersten Wegen, Magen und Darmkanal, wenn auch gerade kein scharfer und reizender, doch aber ein hraftloser Nahrungsstoff abgeschieden wird, sondern auch consecutiv in den zweiten Wegen, des lymphatischen und Drüsensystems, wenn bei schlechter kraftloser Blutbereitung die secernirenden Organe ihre normale Thätigkeit einbüfsen, vor allen andern in der Leber, als dem für die Bereitung und Absonderung der Galle so wichtigen Organe, welches in der Natur dazu bestimmt ist, gewissermafsen das ganze Verdauungsgeschäft zu reguliren und eine kräftige Blutbereitung einzuleiten, da die fehlerhaft bereitete unkräftige Galle es nicht vermag, die im Inhalte des Magens und der dünnen Gedärme vorschlagende Säure zu neutralisiren.

Die unausbleiblichen Folgen dieser ursprünglichen Störungen und regelwidrigen Functionen im ganzen Bereiche der Verdauungsorgane zeigen sich nun primo loco in der Schleimhaut des Darmkanals, als passive Entzündung eines Theils derselben, vorzïglich bein Uebergange der dünnen Gedärme, namentlich am Ende des Ileums, in die dicken Gedärme, bestimmter des Blinddarms und seiner Nachbarschaft, vorzüglich bei typhösen Fiebern, wenn gerade in dieser Gegend des Darmkanals, wegen 
langsamerer peristaltischer Bewegung sich länger verweilt, und die gröfstentheils saure Schärfe den ersten Grund zur Vereiterung der in dieser Gegend besonders in grölserer Zahl gelagerten Schleimdrüsen in Gestalt kleiner Geschwüre in der Schleimhaut der Därme legt.

Ueber diesen letzten Gegenstand insbesondere spricht sich nun $S$ e rtïrner in einer weitläuftigen Kritik einer Abhandlung des Hrn. Dr. Neumann in Berlin, in H ufelands Journal 1827, 31. Stuick abgedruckt, aus. - vid. Annalen etc. 32. Bd. lstes Stück, pag. 14. A. In dieser tief durchdachten Kritik zeigt sich dem unpartbeiischen Leser der prïfend forschende Geist S ertürne $x^{\prime} s$, als dem Vorgange in der Natur angemessen, im vollen Lichte. In Folge dieser von $\mathrm{Neumann}$ aufgestellten Erörterungen über die Natur dieser verderblichen Krankheitserscheinungen nicht nur auf directem Wege im Darmkanal, sondern auch indirect durch Vergiftung des Blutes in den Lungen, durch Knoten- und Tuberkelbildung, bemïht sich Sertürner, den ersten Ursprung dieser Krankheitsformen aus den Producten einer fehlerhaften Verdauung in Magen und Darmkanal herzuleiten, und namentlich durch Ueberladung des Darminhalts mit fressender acider Schärfe zu suchen, wie dies auch höchst wahrscheinlich in manchen Fällen des verderblichen Puerperalfiebers, wenn keine äufsere Luftverderbnifs, als Ursprungsquelle, das Wesen der Krankheit motivirt, supponirt werden möchte, wenn übrigens nur in seltenen Fällen eine active Entzündung unter der Form von Enteritis oder Peritonitis, wie unter andern Brous a is zu erweisen sucht, als zuindende. Potenz anzunehmen ist. Denn wie Sertürner sagt: „örtliche Entzïndungen, Verhärtungen und Verschwärungen etc. im System der Drüsen im Darmkanal und der Lungen etc. sind, wo auch nicht immer, Folgen schädlicher Producte des krankhaften Bildungsgeschäfts, auch wenn wir bei typhösen und synochischen Fibern etc. keine Störung in den ersten Wegen wahrnehmen. Dies sieht man deutlich an dem glücklichen Ausgange, wenn in Fällen dieser Art gleich im ersten Anfange der Krankheit Absorbentien, halbkohlensaurer Kalk, gebrannte Bittererde, mit schleimigten kühlen Mitteln gereicht, und reizende Revellentia äufserlich angewandt werden, auf welche Weise einem örtlichen Zerstörungsprocesse vorgebeugt wird.a

Noch länger bei diesem Gegenstande zu verweilen, dazu ist hier der Ort nicht, der vorurtheilsfreie Leser des wichtigen Gegenstandes wird darin sine ira et odio den scharfprifenden Geist Sertürner's erkennen, um auch hier den Manen des Entschlafenen ein verdientes Todtenopfer zu bringen.

Gleich wichtig, ja vielleicht noch wichtiger, und weiter ausgedehnt, ist aber der Inhalt des 2 . Hefts des 3. Bandes der Annalen, indem der Verf. sich iber mehre gefährliche allgemeine und örtliche Krankheiten, vorzüglich exanthematische, lichtvoll. ausspricht. Scharlach, Masern, Rötheln, Friesel, erysipelatöse Formen aller Art, nebst allen, nicht selten sehr bedeutenden Folgen derselben, sind die Hauptgegenstände der Untersuchungen S ertiirner's, namentlich in Ansehung des grofsen Nutzens der erdigen und alkalischen Heilmittel, durch virifältige Erfahrungen am Krankenbette bestätigt. Vorzigglich ist es das 
Scharlach und die Rose, welchen Sertürner seine Hauptaufmerksamkeit gewidmet hat, und in Beziehung auf erstere Krankheit diesem zweiten Hefte der Annalen den Ausspruch vorgesetzt hat :

"Das Ende der Gefahr beim Scharlach, und den meisten entzündlichen und nervösen örtlichen und allgemeinen Krankheiten, durch neue Entdeckungen der vorzüglichsien Kranhheitsursachen begründet $*$

Aus diesen hier aufgestellten wichtigen Untersuchungen lenchtet doch unbezweifelt das grofse Verdienst Sertürner's hervor, das wissenschaftliche, insbesondere ärztliche Publikum auf Gegenstände aufmerksam gemacht zu haben, die um so mehr die höchste Berücksichtigung verdienen, als sie unmittelbar in das allgemeine Gesundheitswohl der Menschen eingreifen, und, streiten auch hin und wieder die Darstellungen Sertiirner's mit individuellen Meinungen und systematischen Ansichten der Aerzte verschiedener Farben, doch unläugbar die Wahrheit der Sache für sich haben.

War nicht vielleicht in der politisch-wissenschaftlichen Stellung Sertiirner's in der socialen und gelehrten. Welt während seines Lebens die Ursache zu suchen, dafs die wichtigen Entdeckungen, und durch das Zeugnirs Nahestehender bekräftigten Erfahrungen desselben, bislang so wenig Einklang bei Naturforschern und Aerzten fanden, und so wenig zu unpartheiischer Prüfung und Nachahmung des Sertiurner'schen praktischen Verfahrens aufforderten, da man doch in vergangener und gegenwärtiger Zeit so begierig nach neuen Methoden und Heilmitteln, - die Chirurgie aus leicht begreiflichen Gründen etwa ausgenommen - hascht, die weniger einen rationellen Grund und darauf gestützte Frfahrung, als blofs empirischen Gebrauch, auf Symptome basirt, fir sich haben? Wenn Sert iir n e r auch in den schädlichen Producten der Verdauungsorgane und fehlerhafter Mischung des Bluts und der Lymphe die Hauptquelle mehrer der obengenannten, und verschiedener anderer Krankheiten suchte und fand, auch defshalb weder auf chemische Reagentien und Corrigentien, als auf Ausleerung krankhafter materieller Stoffe sein Augenmerk richtete, und glücklich und schnell heilte: so suchen manche seiner Antagonisten mehr ihr Heil in Resolventibus, Eccoproticis, Diaphoreticis etc., ohne die längere Dauer ihrer Heilbemühungen, und eine verlängerte Reconvalescenz zu berïcksichtigen.

Zur Steuer der Wahrheit mufs ich nun bekennen, dafs ich im Vertrauen auf die theoretisch-geprüften, und durch die Erfahrung bestätigten praesumtiven Ansichten Sertürner's, im Laufe mehrer Jahre mein praktisches Verfahren zur Behandlung des Scharlachs, der Masein, Rötheln, des Friesels, der Formen der Rose, z. B. Erysipelas neonatorum, der Blatterrose, wandernden Gesichtsrose, erysipelatöse, rheumatische scrophulöse Augenentzündung, Blepharophthalmia neonatorum, erysipelatöse Hals- und Mandelbräune, Infuenza, allgemein rheumatische Affectionen, zumal wern diese verschiedenen Krankheitszustände rein und unverwickelt erschienen, von vorn herein auf Sertiirner's Ansichten und Fmpfehlung stützte, wo ich dann, vorzüglich bei früh instituirter Behandlung, fast ohne Ausnahme glïckliche 
und schnelle Resultate erhielt, wie ich solches auch insbesondere bei den höchst gefährlichen Formen der Rose, der Blatter- richtiger Blasenrose, und der wandernden Gesichtsrose beobachtete, indem ich stets bemüht war, den gefährlichen Feind auf der Haut fest zu halten, und die acide Rosenschärfe unmittelbar arn loco affecto durch Umschläge von gewärmtem unauflöslichen verbesserten Bleiwasser, oder Bepudern mit pulvis ad erysipelas, beide nach Sertürners Vorschrift hereitet, zu noutralisiren, ohne ein Zuricktreten der giftigen Rosenschärfe nach Innen durch Aufsaugung za fürchten, da selbige durch die Anwendung der äufsern Mittel indifferent gemacht ist. Um nun aber auch eine präsumtive innere Quelle des gefährlichen Uebels zu verstopfen, und einen von daher $z u$ fürchtenden innern Krankheitsprocefs zu verhüten, lasse ich zugleich innerlich die erdig-alkalischen Mittel in grofsen Gaben mit viel warmem Flieder- oder Melissenthee gemischt, reichen, um auch die Contenta des Magens und Darmkanals in möglichst kurzer Zeit zu entsäuren, um zugleich von dieser Seite den allgemeinen Heilprocefs zu fördern. Es gereicht zu meiner vollen Satisfaction, versichern zu können, dafs ich bei dieser Procedur, auch bei den gefährlichsten Rosenformen stets die glücklichsten Erfolge sahe. Dank, öffentlicher Dank meinem verewigten, auch im Grabe unvergefslichen Freunde Sertiurner! -

Noch sei es mir vergönnt, hier eines höchst wichtigen Gegenstandes zu erwähnen, der ebenfalls die Aufmerksamkeit Sertürner's auf sich zog, und welcher mit Einstimmung der Aerzte dem therapeutischen Verfahren nicht selten die grö́fsten Schwierigkeiten darbietet, $1 \mathrm{~m}$ mit Glück den Heilzweck einzuleiten und zu verfolgen. Welchem Arzte ist es nämlich nicht bekannt, wie grofs die Gefahren sind, welche das kaum begonnene Leben junger Kinder bedrohen, und oft unter stürmischen Krankheitserscheinungen den Tod der Kinder veranlassen, wenn es dem Arzte nicht gelingt, in der kürzesten Zeit die erste Ursache dieser gewaltigen Stürme zu beseitigen. In den meisten solcher Falle liegt ohne Zweifel als causa proxima eine vorherrschende Acidität in den Organen der Verdauung zum Grunde der krankhaften Erscheinung, zumal. wenn auch durch vitiöse Function der Leber dem Organismus eine vorstechend gesäuerte Galle zugeführt wird, wie dies die griin gefärbten Darmabgänge zeiģen.

Diese übermächtige Einwirkung einer fressenden sauren Schärfe veranlafst nicht nur die heftigsten örtlichen Leiden im Magen und Darmkanal, durch anhaltendes Schreien sich beurkundende Coliken, Erbrechen und Durchfalle, sondern erregt auch durch die Einwirkung der giftigen sauren Schärfe auf das allgemeine Nervensystem gewaltige Ạsbrüche von Zuckungen und Convulsionen, die man, treffen sie etwa auf die Zeit der Dentition, dem beschwerlichen Zahnausbruche - obwohl höchst wahrscheinlich irrig - zuzuschreiben geneigt ist. Wie lebensgefährlich solche stürmische Zustände der jiingern Kinder sind, und die schnellste Hülfe, vorzüglich durch antacide Mittel, nöthig ist, um die Gefahr abzuwenden, ist übrigens jedem Arzte bekannt.

Es ist ferner physiologisch erwiesen, dafs der kränkliche 
Organismus, zumal je näher er seiner Geburt steht, eine grofse Geneigtheit zu entzündlichen Affectionen aller Art und Formen zeigt, wozu theils eine leider häufig gereichte zu kräftige Nahrung, theils äufsere, das arterielle Blutleben der Kinder mächtig aufregende atmosphärische Einflïsse die Veranlassung geben, wodurch die allgemeine organische Thätigkeit über das natürliche Maafs zu hoch gesteigert wird. Waltet hier zugleich eine vorherrschende positive electrische Spannung der Atmosphäre, bei gleichzeitiger Richtung in $\mathrm{N}, \mathrm{NW}, \mathbf{0}$ und $\mathrm{NO}$, bei geminderter, mit kalten Nebeln begleiteter Luftemperatur vor: so wird durch Einflufs des atmosphärischen Sauerstoffs, durch Uebersäuerung des arteriellen Bluts eingeleitet, und die Entwickelung der entziindlichen Anlage begünstigt. Entzündliche Brust -, Hals- und Gehirnaffectionen, - wozu Sertiirner mit Recht auch den Croup rechnet, sind dann an der Ordnung des Tages, und raffen manches hart ergriffene Kind dahin.

Auch diese gefährlichen krankhaften Zustände der Kinder eignen sich nach Sertürner's Beobachtung und Erfahrung ganz für die rasche Anwendung erdig-alkalischer Mittel, wodurch dann die saure giftige Schärfe im Organismus der Kinder auf dem kürzesten Wege abgestumpft, und der Sturm besänftigt wird.

Bevor ich aber den Manen meines entschlafenen Freundes einen letzten Nachruf als würdiges Todtenopfer darbringe, glaube ich noch eines höchst wichtigen verhängnifsvollen Ereignisses erwähnen zu müssen, wo eine impetuöse, für allgemeines Menschenwohl die grölsten unabwendbaren Gefahren drohende Zeit unsern Sertirne $r$ dahin bestimmte, um auch von seiner Seite öffentlich sich mit Vorschlägen und Vermuthungen an das gesammte europäische ärztliche Publikum zu wenden, und dasselbe zu Rathe und möglicher Hülfe gegen einen Feind aufzufordern, der in gräfsliches Dunkel verborgen, allen Versuchen der heilenden Kunst trotzte, und ohne Unterschied des Standes, Alters und Geschlechts tödtend und würgend das Leben der Menschen tilgte, und sogar die Thiere nicht verschonte.

Bereits im 2. Hefte des 2. Bandes der Annalen sprach sich Sertürner über die entzündlichen und nervösen Fieber der heifsen Klimate aus, und suchte ihren gastrischen Ursprung nachzuweisen, und zugleich auf den englischen Arzt Henders on gestützt, seine Vermuthung wegen des Ursprungs der schrecklichen epidemischen Cholera aufzustellen, dafs auch bei dieser fïrchterlichen Krankheit die erdig-alkalischen Mittel ihren grofsen ausgezeichneten Nutzen gewähren möchten, da auch die anglo-indischen Aerzte bei der dortigen Krankheit die grofse Wirksamkeit der Magnesia, aufser grofsen Gaben von Calomel bestätigten, indem sie mit Henderson die Ursache der Cholera von einer, im Magen und Darmkanal abgelagerten scharfen Säure ableiteten. Bei dem Gebrauch von Magnesia, Ricinusöl und Laudanum, will übrigens Henderson keinen Kranken verloren haben. Aber die Beobachtungen desselben bezogen sich nur auf die gewöhnliche sporadische herbstliche Cholera in Hindostan, die von dem häufigen Genusse säuerlicher 
Früchte, Melonen, Ananas, Trauben etc. auszubrechen pflegt, wenn man dabei keinen Arrac trinkt.

Ganz anders verhielt sich aber die Sache bei der im Jahre 1817 in Hindostan ausgebrochenen epidemisch-wandernden Cholera, die auf ihren mörderischen Zügen imLaufe der folgenden Jahre die europäischen Grenzen erreichte, und von da an seit 1831 und in den folgenden Jahren ganz Europa, wenige Iänder ausgenommen, so wie auch Nordamerika mit ihrer gräfslichen Todesfackel iberzog, und allen Anstrengungen der problematisch sichern heilenden Kunst Trotz bot. Als nun diese furchtbare, in ihrem innern Wesen räthselhafte und unerklärte Seuche unserm Lande sich immer mehr nähern zu wollen schien, so trat Sertürner mit seiner Ansicht über die vermuthliche ursachliche Genesis derselben öffentlich auf, und urgirte namentlich eine saure Grundlage des verderblichen Zïindstoffs, gestiitzt auf die Untersuchung und Erfahrung Hermann's in Moskau, Dann's in Danzig, und $E$ is en mann's in Würzburg, denen gemäfs alle Auswürfe des IJarmkanals nach Oben und U11ten sauer reagirten, und das wenige Blutserum freie Essigsäure enthielt. Er that dieserhalb den Vorschlag, dem Winke der Natur zu folgen, und die furchtbare Seuche, als Haupterfordernifs der Behandlung mit erdig-alkalischen Mitteln in grofsen Gaben zu bekämpfen, zugleich auch, nach Art der anglo-indischen Aerzte, Colomel und Opium, beide in grolsen Gaben, und Ricinusöl damit zu verbinden.

Er liefs nun auf seine Kosten zwei kleine Schriften drukken, unter folgenden Titeln:

1) Dringende Aufforderung an das deutsche Vaterland, in $\boldsymbol{B e}$ ziehung der orientalischen Brechruler. Gött. 1831.

2) Weitere Entwicklung der neuen zuversichtlichen Schutzmethode gegen die Cholera, und der Ansicht über ihren höchst wahrscheinlichen Ursprung. Gött. 1831.

Leider aber fanden die Vorschläge Sertiurner's bei den betheiligten Aerzten iberall keinen Eingang, und unter der Fluth der von allen Seiten auftauchenden Choleraschriften fand sich nirgends eine Spur von der Beachtung der theoretischen Ansichten und Vorschläge desselben. Gliicklicher Weise wurde nun aber unser Land grörsenthejls von der Krankheit verschont, wefshalb S e rtiirner keine Gelegenheit fand, sich selbst von der Realität seiner Vorschläge praßisch zu überzeugen. Es wäre zu wïnschen gewesen, Sertürner hätte Muth, Entschlossenheit und Selbstvertrauen gehabt, sich an Orten der Infection, Hamburg etwa, von der Zuverlässigkeit seiner Ansichten praktisch zu überzeugen, um im erfolgreichen Falle Tausende und aber Tausende vom sichern Choleratode zu retten. Welcher Lohn wïrde hinreichend gewesen sein, um das Andenken des entschlossenen Menschenretters für Gegenwart und Zukunft nach Verdienst zu wïrdigen? -

Möge endlich, - dies sei mein letztes Wort! - das Obige hinreichen, als Panegyricus den Manen meines unvergefslichen Freundes ein bleibendes Andenken seines vielseitigen Verdienstes um die Wissenschaft, auch im Tode zu erhalten, den fürderhin die Ruhe im Grabe für alle Widerwärtigkeiten im Leben entschädigt. 
Möge diesem meinem Nachrufe ein bleibender Nachruhm des Verewigten nicht fehlen! Fiir den Augenblick genüge mir der Ausspruch Göthe's: "Liebreiches, ehrenwerthes Andenken ist alles, was wir dem geliebten Todten zu gehen vermögen.*)

\section{2) Vereinsangelegenheiten.}

\section{Directorialconferenz zu Lemgo am 7. März 1842.}

1) In Betreff der Abrechnung eines Kreises von 1842 fand sich das Directorium, auf Anzeige der Direction der Generalkasse, zu der Erinnerung genöthigt, dafs, da die Ausgaben für diesen Kreis dessen Einnahme bedeutend übersteigen, dieser Uebelstand fuir die Folge nicht wieder vorkomme, weil es sonst pine reine Unmöglichkeit ist, dafs Einnahme und Ausgabe je in Gleichgewicht kommen. Der Inhalt der Statuten muls in diesem Puncte genau beachtet werden, und kann für die Folge eine dieses nicht beachtende Abrechnung nicht mehr angenommen werden.

2) In Betreff der Bucholz-Gehlen-Trommsdorff'schen Stiftung wurde ein Bericht von Hrn. Vicedir. Bucholz in Erfurt vorgelegt, betreffend das dieser Stiftung vom Hrn. Geh. Medicinalrath Dr. Fis che $r$ in Erfurt zugesagte Legat. Der menschenfreundliche Wohlthäter hat dem Vorstande der Stiftung in Erfurt einen Revers ibergeben, nach welchem derselben nach dem Tode des Hrn. Geh. Medicinalraths $F$ ischer und seiner Gattin das testamentarisch vermachte Legat von 100 ThIr. ausgezahlt werden soll.

3) Der Bucholz-Gehlen-Trommsdorff'schen Stiftung wurden 56 Thlr, übergeben, die vom Hrn. Prof. Dr. Erdmann in Leipzig von mehren dortigen Drogueriehandlungen für die edlen $\mathbf{Z}$ wecke der Stiftung eingegangen waren.

4) Desgl. wurden gedachter Stiftung noch 35 Thlr. übergeben, die Hr. Vicedir. Dr. M e u r e r eingesandt, die von mehren Herren Gehülfen aus Dresden und der Umgegend eingegangen waren.

5) Den würdigen invaliden Gehülfen, den Herren Hum mel und $\mathrm{K}$ arbe, wurde die von Hrn. Viced. Bolle in Angermünde nachgesuchte Unterstützung auch für das laufende Jahr bewilligt.

*) Für diese Schilderung des Wirkens unsers entschlafenen Freundes, Sertürner, vornehmlich im Gebiete der Medicin, bin ich Hrn. Dr. Nolte recht sehr verbunden. Gleich nach dem Tode des geistvollen, mir innig befreundeten Mannes wünschte ich sein Andenken durch ein biographisches Denkmal in unserer Zeitschrift zu feiern, und erbat mir delshalb dazu die nöthigen biographischen Nachrichten, die mir auch zugesagt wurden, mir aber noch nicht geworden sind. Sobald solche mir zugehen, werde ich es als eine heilige Pflicht betrachten, diese Schuld der Dankbarkeit den Manen Sertürner's abzutragen.

\section{Brandes.}

